\title{
Norbert Schloßmacher (dir.), Beethoven. Die Bonner Jahre.
}

\section{Héloïse Elisabeth Ducatteau}

\section{OpenEdition \\ Journals}

Electronic version

URL: https://journals.openedition.org/ifha/11603

DOI: 10.4000/ifha.11603

ISSN: 2198-8943

\section{Publisher}

IFRA - Institut franco-allemand (sciences historiques et sociales)

Electronic reference

Héloïse Elisabeth Ducatteau, "Norbert Schloßmacher (dir.), Beethoven. Die Bonner Jahre.", Revue de I'IFHA [Online], Date of review, Online since 16 January 2022, connection on 18 January 2022. URL: http:// journals.openedition.org/ifha/17603; DOI: https://doi.org/10.4000/ifha.17603

This text was automatically generated on 18 January 2022

(CIFHA 


\section{Norbert Schloßmacher (dir.), Beethoven. Die Bonner Jahre.}

Héloïse Elisabeth Ducatteau

\section{REFERENCES}

Norbert Schloßmacher (dir.), Beethoven. Die Bonner Jahre, Köln: Böhlau Verlag, 2020, 560 p, open access. https://doi.org/10.7788/9783412519704 


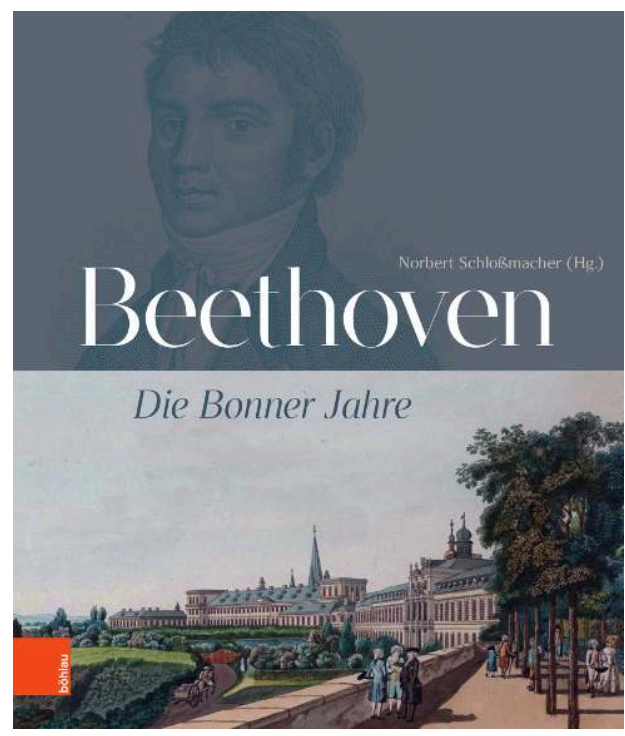

C'est l'anniversaire des 150 ans de la naissance du compositeur Beethoven qui a motivé la rédaction du présent ouvrage. Norbert Schloßmacher part du principe que ce génie des partitions est le plus connu. On pourra contester cet avis qui n'est d'ailleurs pas vérifié sociologiquement. Si le Bonnois devenu sourd a pu certes être popularisé par la série de films américains Beethoven à partir de 1992, Mozart n'a-t-il pas réussi à s'attirer un nouveau public grâce à Mozart, l'opéra rock produit en 2009 puis grâce à la série américaine Mozart in the Jungle ? Quoi qu'il en soit, on s'accordera sur le fait que Beethoven dépasse le cadre musicologique et mérite toute l'attention des historiens du social. Bien que les questions qui se posent ne soient pas dans l'ensemble nouvelles, des sources jusqu'ici non explorées le sont enfin. Il s'agit de mieux comprendre l'environnement bonnois dans lequel a évolué le jeune musicien entre 1770 et 1792, année de son départ définitif à Vienne où il meurt en 1827. De quelle façon a-t-il pu présider à l'émergence de son talent ? Par années bonnnoises, notre historien intègre aussi Godesberg. Cette source thermale est découverte en 1790 et ne sera rattachée à la ville de Bonn qu'en 1969. Très vite, cette station thermale devient une résidence sécondaire de la cour et c'est là que Beethoven fait la connaissance de Josef Haydn avant son déménagement à Vienne.

Dans la première partie, "Les débuts ", Silke Bettermann s'intéresse à sa famille tandis que le directeur de la publication présente son baptême. Gisbert Knopp fait un pas de côté et rentre dans l'histoire architecturale de l'église concernée, Saint Rémi (Remigius en allemand).

Sortant du cadre privé, la deuxième partie, "Les prérequis ", traite du contexte socioculturel à Bonn dans la deuxième moitié du XVIII ${ }^{e}$ siècle. Alexander Wolfshohl a méticuleusement passé en revue les catalogues de bibliothèques, les répertoires des emprunts, les listes des inscrits. Il en ressort que les femmes étaient assez éclairées et pouvaient s'adonner à la lecture dans une bonne proportion pour l'époque. Certains érudits pouvaient se retrouver au sein d'un club de lecture (la Lesegesellschaft) ou des francs-maçons. La haute école (Hohe Schule) témoigne du rôle-clé de Bonn au niveau des Lumières. Le cercle plus informel du Zehrgarten comptait parmi se membres le professeur de droit naturel et des peuples Bartholomäus Fischenich. Michael Ladenburger explique le développement rapide d'instruments à clavier du temps de 
Beethoven avant de s'attarder sur le facteur de pianos et d'orgues qui vécut à Bonn, Gottlieb Friedrich Riedler.

La troisième partie, "Contemporains, amis, compagnons de route ", aurait logiquement dû adopter l'écriture inclusive puisqu'il est question d'une ses amies d'enfance, Eleonore von Breuning. Cette dernière épousa un ami d'enfance du musicien, Franz Gerhard Wegeler, devenu médecin. Beethoven n'entretint pas toujours un rapport pacifique avec celui-ci. De même, sa relation avec son éditeur bonnois, Nikolaus Simrock, se montra ambivalente. Il est assez naturellement question de la famille Ries étant donné que Franz Anton Ries, le père, était professeur de violon du jeune Ludwig. Un autre professeur de Beethoven est passé à la loupe : Christian Gottlob Neefe dont le rôle est ici relativisé. Pia Hecke est la première à faire le lien entre la Musique pour un bal de chevaliers (Musik zu einem Ritterballett) et le comte Ferdinand Ernst von Waldstein, l'un de ses mécènes. C'est lui qui surnomma son jeune ami lors de sa migration viennoise esprit de Beethoven avec les mains de Haydn.

Yvonne Leiverkus rend compte dans son article de la postérité de Beethoven à Bonn. Alors qu'il est installé à Vienne, son œuvre est jouée, parfois pour la première fois, à Bonn par le fils Ferdinand Ries mais aussi sous la houlette de Heinrich Karl Breidenstein, le premier détenteur d'une chaire de musicologie en Allemagne. On lui doit la première fête de Beethoven en 1845 lors de laquelle sont interprétées pour la première fois la Missa solemnis et la Neuvième Symphonie. Étonnamment, le décès de Beethoven a été annoncé assez tardivement à Bonn, plus de deux mois après, ce qui n'a bien sûr pas empêché que les funérailles aient lieu en bonne et due forme par sa ville nourricière. Inévitablement, l'autrice fait mention de l'érection de la statue du musicien sur la place de la cathédrale sans oublier la création de la place Beethoven.

\section{INDEX}

Subjects: Histoire de la culture, Histoire de l'art, Histoire des femmes

Chronological index: Époque moderne

\section{AUTHOR}

\section{HÉLOÏSE ELISABETH DUCATTEAU}

Sciences Po Paris (campus de Nancy) 